\title{
TEMA 11-2016: Síndrome de Ovario Poliquistico
}

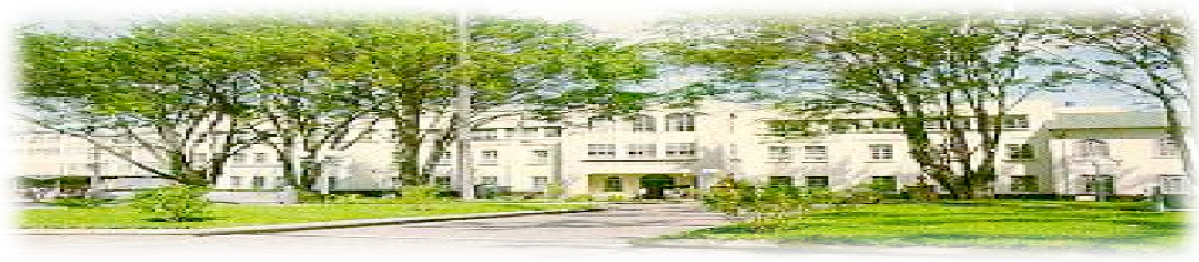

Hospital San Juan de Dios, San José, Costa Rica. Fundado en 1845

$\begin{array}{ll}\text { Recibido: } & 28 / 12 / 2015 \\ \text { Aceptado: } & 07 / 01 / 2016\end{array}$

María Fernanda Borbón Cordero ${ }^{1}$ Fernán Robles Quirós ${ }^{2}$

${ }^{1}$ Médico General, EBAIS La Carpio, Clínica Bíblica, correo electrónico: maferborbon@gmail.com

${ }^{2}$ Médico Especialista en Ginecología y Obstetricia, Hospital México, Caja Costarricense de Seguro Social

\section{RESUMEN}

El síndrome de ovario poliquístico afecta $5 \%$ a $10 \%$ de las mujeres en edad fértil. Es un síndrome heterogéneo caracterizado por anovulación crónica hiperandrogénica, secundaria a una disfunción ovárica intrínseca. Aunque las pacientes afectadas se ven muy perturbadas por las manifestaciones cutáneas, que incluyen acné, hirsutismo, alopecia y acantosis nigricans ${ }^{(1-2)}$, el síndrome de ovario poliquístico puede tener implicaciones mucho más serias que las encontradas en la piel; con frecuencia causa irregularidades menstruales e infertilidad y, además, está agravado debido a una hiperinsulinemia por resistencia a la insulina, con el consecuente riesgo de diabetes mellitus y síndrome metabólico. Dentro de los factores más importantes de su abordaje es la disminución de peso, así como determinar el deseo de concebir de una paciente, a partir de dichos puntos se decide su tratamiento.

\section{PALABRAS CLAVE}

Síndrome de ovario poliquístico, acné, hirsutismo, resistencia a la insulina, trastornos menstruales, alopecia androgénica

\section{ABSTRACT}

Polycystic ovary syndrome affects $5 \%$ to $10 \%$ of reproductive-age women and it is one of the most common endocrine disorders in women. It is a heterogeneous syndrome of hyperandrogenic anovulation that is typically due to intrinsic ovarian dysfunction. Although patients affected are often very disturbed by the cutaneous manifestations, including acne, hirsutism, alopecia and acanthosis nigricans, the clinical manifestations of polycystic ovary syndrome ramify far beyond the skin. Polycystic ovary syndrome frequently causes menstrual abnormalities and infertility. This syndrome is often aggravated by insulinresistant hyperinsulinemia with its risks of diabetes mellitus and metabolic syndrome and their complications. Among the most important factors in 
their approach is the reduction in weight as well as the desire to conceive, treatment is decided based on these points.

\section{KEY WORDS}

Polycystic ovary syndrome, acne, hirsutism, insulin resistance, menstrual irregularity, pattern alopecia.

\section{INTRODUCCIÓN}

El hiperandrogenismo, o producción excesiva de andrógenos, es un trastorno que afecta, principalmente, al sexo femenino. Esta condición puede estar asociada a diferentes enfermedades, y la más frecuente es el síndrome de ovarios poliquísticos que explica, aproximadamente, $82 \%$ de los casos de hiperandrogenismo en mujeres. Desde abril de 1990 se ha venido utilizando la definición del National Institute of Health de Bethesda (EE.UU.) ${ }^{(3)}$, basada en disfunción menstrual (oligo/anovulación), la presencia clínica de hiperandrogenismo (hirsutismo, acné y alopecia androgénica) o niveles de andrógenos elevados en sangre, excluidas otras alteraciones hormonales (hiperprolactinemia, hiperplasia suprarrenal no clásica y trastornos tiroideos), sin contemplar en ningún momento la apariencia ecográfica de los ovarios.

\section{DISCUSIÓN}

En mayo de 2003 la reunión de expertos de Rotterdam1 establece los criterios diagnósticos vigentes en la actualidad, en los que la imagen ecográfica cobra de nuevo valor.

Criterios diagnósticos del síndrome de ovarios poliquísticos (criterios de Rotterdam 2003. ESHRE/ASRM) ${ }^{(4)}$ :

- Presencia de oligo/anovulación

- Signos clínicos y/o bioquímicos de hiperandrogenismo

- Ovarios de apariencia poliquística en la ecografía. (Se exige al

menos alguno de estos criterios:

12 o más folículos de 2 a $9 \mathrm{~mm}$

de diámetro y/o volumen ovárico superior a 10

cc)
- La presencia de dos de los tres criterios establecidos es suficiente

para el diagnóstico del SOP

Clínica:

Los elementos clínicos más importantes del SOP son:

Hirsutismo: Aparece en un $70 \%$ de las mujeres con $\mathrm{SOP}^{(5)}$. Es un indicador clínico del exceso de andrógenos e hiperinsulinismo. Aunque la testosterona total no aparezca elevada la libre sí lo está, debido a la disminución de la proteína transportadora. Hay que distinguir el hirsutismo, presencia excesiva devello en las zonas andrógeno dependientes, de la hipertricosis, aumento de vello en otras zonas no necesariamente debida a la acción androgénica. En el hirsutismo idiopático los niveles de andrógenos son normales, pero hay una excesiva actividad periférica de la $5-\alpha$ reductasa o una sensibilidad exagerada por parte del receptor androgénico. Para valorar el hirsutismo se recomienda utilizar la escala semicuantitativa de Ferriman-Gallwey ${ }^{(6)}$.

Alteraciones menstruales: En un 70\% aparece ritmo menstrual irregular, con oligomenorrea o amenorrea, aunque no es imprescindible su presencia para el diagnóstico. Podemos encontrar hasta un $30 \%$ de las pacientes con función ovulatoria normal. La oligomenorreamantenida en la adolescencia en un elevado porcentaje se asocia a futuro SOP. Cuando la oligoanovulación va ligada a la obesidad es muy beneficiosa la disminución de peso para mejorarla y aumentar la fertilidad.

Acné: Afecta a un tercio de las mujeres con SOP. No se ha constatado que sea precisa una elevación de los andrógenos en sangre para su aparición.

Obesidad: Un $30-35 \%$ presenta aumento significativo del índice de masa corporal y del índice cintura- ${ }^{\text {cadera(6) }}$. La resistencia a la insulina y la hiperinsulinemia compensadora en mujeres con SOP aparecen tanto en mujeres obesas como en las delgadas, aunque se ve aumentada por la obesidad. La obesidad por sí misma produce alteraciones del ciclo sin SOP, pero cuando hay SOP las alteraciones menstruales son más intensas. 
Complicaciones en el embarazo: Se han descrito varias. El aborto recurrente es controvertido, aunque la balanza se inclina a favor de la significación. La diabetes gestacional se ha encontrado asociada con significación estadística al SOP. La preeclampsia se ha descrito con mayor incidencia en algunos estudios, pero no en otros.

Aumento del riesgo cardiovascular: Un 30-35\% de los pacientes con SOP presentan intolerancia a la glucosa, con mayor tasa de progresión a la diabetes franca. El riesgo de desarrollar una diabetes mellitus tipo 2 en el SOP es de 3 a 7 veces mayor que el de la población general ${ }^{(7)}$. Un $27 \%$ de las mujeres premenopáusicas diagnosticadas de diabetes mellitus 2 han sido diagnosticadas de SOP. También se ha encontrado una mayor incidencia de hipertensión arterial y de hiperlipemia en el SOP. Todo lo anterior puede condicionar un mayor riesgo de cardiopatía isquémica en las pacientes con SOP. La incidencia de ictus parece estar ligeramente elevada.

Hiperplasia y cáncer de endometrio: Se ha supuesto que la secreción continua de estrógenos no compensada con progesterona es un factor de riesgo de cáncer de endometrio en las pacientes con SOP, pero la evidencia es limitada.

Hiperprolactinemia: Muchas pacientes con hiperandrogenismo pueden tener niveles de prolactina en los límites superiores de la normalidad o ligeramente superiores. Niveles elevados de Prolactina inducen intolerancia a la glucosa, hiperinsulinemia y Resistencia a la insulina.

Acantosis nigricans: Clínicamente se caracteriza por placas de bordes mal definidos, café- grisáceas, de superficie afelpada y con acentuación de las marcas cutáneas localizadas en la región postero-lateral del cuello, axilas, ingle y región inframamaria. Se considera un marcador de resistencia a la insulina $y$, aunque puede estar asociada a neoplasias, medicamentos como el ácido nicotínico o ciertos síndromes genéticos, lo más frecuente es que se presente en pacientes con obesidad e hiperinsulinemia.

\section{Diagnostico:}

No existen datos patognomónicos del SOP. La historia clínica general y gineco-obstétrica, la exploración física, algunas determinaciones de laboratorio y la ecografía serán las bases para poder aplicar los vigentes criterios diagnósticos de Rotterdam y excluir otras patologías. La exploración física permite detectar el hirsutismo, acné, alopecia androgénica $u$ otros signos de virilización. No obstante, hay que tener en cuenta el factor de confusión que pueden suponer las variaciones étnicas, la aplicación de tratamiento cosmético, depilaciones, etc. También hay que minimizar la subjetividad del médico a la hora de valorar estos hallazgos, utilizando en lo posible escalas semicuantitativas (Ferriman-Gallwey para el hirsutismo ${ }^{(8)}$. Presencia de vello en labio superior, barba, abdomen inferior y piernas. Debe estimarse tanto el índice de masa corporal, como el perímetro abdomen/cadera. Debe valorarse la existencia e intensidad de acné y buscar lesiones hiperpigmentadas aterciopeladas en axilas, nuca, cuello, zonas submamarias y otros pliegues, que podrían sugerir Acanthosis Nigricans. Debe realizarse una toma de la presión arterial. El médico de familia no debe olvidar realizar un exámen pélvico de estas pacientes. La mayoría de los autores aconsejan la determinación de testosterona libre. Un $50 \%$ de los SOP presentan una elevación de la $\mathrm{LH}$, y tradicionalmente se usó el cociente LH-FSH $>2$ como criterio diagnóstico ${ }^{(9)}$.

Ecografia ovárica: Los ovarios de apariencia poliquística son motivo de falsos positivos. También hay falsos negativos, ya que un $30 \%$ de las mujeres presentan ovarios de apariencia normal. Las mujeres con ovarios de apariencia poliquística, sin SOP, tienen una mayor frecuencia de hiperandrogenismo, infertilidad y abortos. Sólo es necesario que los criterios se cumplan en uno de los dos ovarios. No son aplicables a las mujeres que toman anticonceptivos.

En estas pacientes es muy importante descartar otras causas de oligoanovulación y de virilización: hiperprolactinemia, amenorreas hipotalámicas, fallos ováricos primarios, disfunción tiroidea. La

determinación de prolactina, FSH, LH y TSH puede estar indicada. La prueba de la progesterona puede ayudar en el estudio de la amenorrea (en el SOP aparece un sangrado tras la toma). También otras causas de hirsutismo, acné.

Tratamiento:

Lo primero que debemos plantear a estas pacientes es la pérdida de peso y el ejercicio, sobre todo en las obesas. La reducción de peso provoca la normalización de la Resistencia a la Insulina y 
del metabolismo de las gonadotropinas, se restauran los ciclos ovulatorios y se regularizan los andrógenos. La siguiente consideración es valorar si la paciente tiene deseo de concebir. Cuando no hay deseo de concebir, el tratamiento ha de ir encaminado a aliviar la sintomatología. Los anticonceptivos hormonales combinados han demostrado en estas mujeres una disminución de los niveles de DHEAS del orden del 30 al 60\% y mejoran el acné e hirsutismo mientras se realiza el tratamiento. Debemos elegir progestágenos que no tengan acción androgénica, preferiblemente

Acetato de ciproterona o drospirenona. Se puede utilizar la espironolactona como antiandrógeno, a dosis de 25- $400 \mathrm{mg}$, habiendo demostrado una dosis de $100 \mathrm{mg}$ diarios una reducción del hirsutismo del $70-75 \%{ }^{(10)}$.

Si la paciente desea concebir, tras la dieta y el ejercicio lo siguiente sería la inducción a la ovulación. Hay experiencia en el uso de clomifeno por médicos de familia en este tipo de paciente, una vez excluidas otras causas de infertilidad. Se demostró que la tasa de fertilización era mejor en mujeres a las que, además del clomifeno, se les administra metformina. Muchos casos precisarán derivación a ginecología y la aplicación de técnicas de reproducción asistida. Se debe derivar a una unidad de reproducción a las mujeres con un SOP bien controlado a los 6 meses de esterilidad.

\section{CONCLUSIONES}

El síndrome de ovario poliquistico es una disfunción endocrino-metabólica altamente prevalente que se ve en un 5 a $10 \%$ de las mujeres en edad reproductiva, de presentación muy variable, cuyas manifestaciones más frecuentes son las alteraciones menstruales, hiperandrogenismo y obesidad. La insulino resistencia juega un rol central en el desarrollo del síndrome en un grupo significativo de pacientes, y debe ser estudiada y manejada adecuadamente para lograr una terapia exitosa. Los cambios de estilo de vida, consistentes principalmente en dieta y ejercicios deben ser incentivados permanentemente en las pacientes con Síndrome de ovario poliquistico, ya que sus efectos no sólo son útiles para corregir alteraciones en lo inmediato sino también son fundamentales en la prevención de patología crónica como diabetes mellitus 2 y enfermedad cardiovascular

\section{BIBLIOGRAFÍA}

1. Ermaanh D. Polycystic ovary syndrome review. N Engl J Med. 2005;352:122336.

2. Guzick DS Wing R Smith D Berga SL Winters SJ. Endocrine consequences of weight loss in obese, hyperandrogenic, anovulatory women. Fertil Steril 1994;61:598-604

3. King J. Polycystyc ovary syndrome. J Midwifery Womens Health. 2006;51:415-22.

4. The Rotterdam ESHRE/ASRMsponsered PCOS consensus workshop group. Revised 2003 consensus on diagnostic criteria and long-term health risks related to polycystic ovary syndrome. Hum Reprod. 2004;19:41-7.

5. Chang R. A practical approach to the diagnosis of polycystic ovary syndrome. Am J Obstet Gynecol. 2004;191:713-7.

6. Brewer M Pawelczak M Kessler M Shah B. A review of polycystic ovarian syndrome in adolescents. Minerva Pediatr. 2010;62:459-73.

7. Jonard S Roberty Y Cortet-Rudelli C Pigny P Decantec D. Ultrasound examination in polycystic ovary syndrome. Is it worth to count follicles? Hum Reprod. 2003;18:598-603.

8. Nam MM Strauss JF. Genetics of polycystic ovarian syndrome. Clin Obstet Gynecol. 2007;50:188-204

9. Rosenfield RL. What every physician should know about polycystic ovary syndrome. Dermatol Ther. 2008;21:35461.

10. Stein I Leventhal M. Amenorrhoea associated with bilateral polycystic ovaries. Am J Obstet Gynecol. 1935;29:181-5.

\section{CONFLICTOS DE INTERES}

Los autores declaran que no existe ningún conflicto de interés. 